\title{
Días de Radio. El esfuerzo hacia la recuperación: la radio como integración
}

\author{
Itzíar Martínez González \\ Óscar Pérez López \\ Francisco Javier Bueno Roca \\ Manuel Jiménez Martín \\ Antonia Ruiz del Campo \\ Guillermo Segura Rodríguez \\ Juan Francisco Sigüenza Encabo \\ Centro de Rehabilitación Psicosocial Los Cármenes. Madrid.
}

\begin{abstract}
Resumen
Desde el Centro de Rehabilitación Psicosocial (“CRPS”) Los Cármenes (inscrito dentro de la Red Pública de Atención Social para Personas con Enfermedad Mental Grave y Duradera, dependiente de la Consejería de Familia y Asuntos Sociales de la Comunidad de Madrid (www.comunidad.org); gestionado por Instituto de Trabajo Social y Servicios Sociales (INTRESS), se desarrolla desde el 2004 un proyecto que consiste en la realización del programa de radio comunitaria "Ábrete Camino". La metodología, basada en los valores clave de la recuperación, permite que los/as participantes sean cada vez más protagonistas, tanto en el programa como en sus vidas. Ellos/as mismos/as nos cuentan su experiencia a través de un debate en directo en una de sus emisiones. Valoran la experiencia como muy positiva, siendo una actividad significativa en su proyecto vital.
\end{abstract}

Palabras claves: recuperación, integración comunitaria, salud mental.

\begin{abstract}
Radio Days. The effort to recovery: the radio as integration. From the Center for Psychosocial Rehabilitation ("CRPS") Los Cármenes (registered in the Public Network of Social Care for People with Severe and Enduring Mental Illness, under the Ministry of Family and Social Affairs of the Community of Madrid, operated by INTRESS ), developed since 2004 a project that consists in making community radio program "Ábrete Camino". The methodology, based on the core values of the recovery, allowing the participants to be increasingly more players in both the program and in their lives. They have experience through a live debate in one of their broadcasts.
\end{abstract}

Keywords: recovery; community integration; mental illness.

$\mathrm{E}$ 1 programa de radio "Ábrete Camino" parte desde el Centro de Rehabilitación Psicosocial "Los Cármenes" ("CRPS"), en la ciudad de Madrid. Este centro se inscribe dentro de la Red Pública de Atención Social para Personas con Enfermedad Mental Grave y Duradera, dependiente de la Consejería de Familia y Asuntos Sociales de la Comunidad de Madrid ${ }^{1}$, siendo gestionado desde su inicio por INTRESS (Instituto de Trabajo Social y Servicios Sociales). Los objetivos principales de este recurso son, desarrollar Programas de Rehabilitación para las personas con enfermedad mental duradera, con el fin de recuperar la autonomía personal. Además, presta asesoramiento y soporte a las familias. Por último, ofrece apoyo social a las personas que lo necesiten para mantenerse dentro de su comunidad, y para que aumenten sus redes sociales.
Por tanto, nuestro centro tiene un carácter recuperador, buscando que sea la propia persona con una enfermedad mental la que pueda gestionar autónomamente su propia vida. El objetivo de este trabajo es mostrar de primera mano la experiencia que la participación en un programa de radio ofrece en los procesos de recuperación a personas con enfermedad mental.

\section{La Radio y sus objetivos}

Ábrete Camino comienza su andadura en otoño de 2004, tras unos meses de preparativos, en los que se realizó un entrenamiento en pronunciación, redacción, etc., y llega a la sexta temporada de emisión con sus locutores/as más protagonistas que nunca. Más de 30 usuarios/as han participado en esta experiencia a lo largo de los años. En total hablamos de más de 200 programas 
de una hora de duración cada uno. Nuestra filosofía ha sido emitir desde emisoras no vinculadas a organismos oficiales; son las llamadas Radios Libres. En la actualidad trabajamos con una emisora desde un barrio popular de Madrid (Villaverde) que permite que el programa se oiga en directo, tanto por el dial como por internet, así como que pueda ser grabado para colgarlo en el blog semanalmente.

Los mismos/as locutores/as eligieron desde el principio el formato del programa; es un magazine compuesto por distintas secciones, tales como: comentarios, sociedad, deportes, ciencia, medio ambiente, cine, música, cocina, cultura. Los últimos 20 25 minutos se reservan para nuestra sección estrella: el debate, moderado por uno/a de los/as locutores/as, en el que se discuten temas de todo tipo, con total libertad de opinión. Con cierta frecuencia, el debate se ve sustituido por la posibilidad de realizar una entrevista. A más de una cuarta parte de nuestros programas han acudido invitados/as: psiquiatras, cantautores, fotógrafos, pintores, poetisas, psicólogos/as, usuarios/as de otros recursos, científicos, técnicos/as de distintas profesiones, estudiantes.

No podemos continuar sin pararnos en las distintas proyecciones que el programa ha tenido en todo este tiempo. Por un lado, diversos medios de comunicación (cadenas nacionales de radio y televisión, cadenas locales de televisión, periódicos, revistas especializadas, páginas web divulgativas), se han interesado por el programa y nos han entrevistado. Por otro lado, en los dos últimos años, han sido otros recursos de acción social los que han aprovechado nuestras posibilidades como promotores de la lucha contra el estigma, con el objetivo de hacer oír su voz. Paralelamente, los/as locutores/as han sido ponentes en diversas jornadas y mesas redondas relacionadas con la recuperación psicosocial, la integración social y la lucha contra el estigma.

Nuestra metodología está basada en los siguientes valores clave de recuperación (Farkas, 2007):

a. Orientación hacia la persona: Significa tener una visión holística de la persona, ver su "biografía entera", dar más importancia a las potencialidades que a lo psicopatológico, dar importancia a la historia personal de los/as participantes, a lo que aporta cada uno de ellos. Centrarse en talentos y recursos personales, validando su participación en el programa.

b. Implicación de la persona: Prestamos apoyo y herramientas para que sean las personas las que puedan acabar planificando la actividad en función de sus intereses. El formato del programa de Radio involucra a las personas asistentes al mismo en su planificación, preparación, la toma de decisiones y en general la mayor autonomía en la realización de la actividad. De un modo progresivo, se trabaja en la toma de decisiones autónoma, las actividades que previamente hacían los/as profesionales, van recayendo en los/as participantes, para finalmente recaer todo el peso del programa en la gestión autónoma por parte de los participantes en el mismo, sin el apoyo o supervisión de los profesionales.

c. Potenciar la autodeterminación: Es una actividad dirigida al disfrute, la creatividad, la expresión de ideas, el contacto con otras personas, donde se deja a las personas tomar sus propias decisiones, sin coacción, colaborando y fomentando el sentido de empoderamiento (empowerment) y de identidad propia.
Ayudando a la persona en retomar el significado y propósito en sus vidas.

d. Esperanza/Potencial para crecer: La visión en "Ábrete Camino" es incluir a los/as participantes en todos los aspectos relacionados con su funcionamiento: no sólo nos referimos al programa en sí, sino a proyectos de futuro, gestiones administrativas, captación de nuevos/as miembros, modificaciones varias, etc. Son los/as protagonistas y el motor del programa, tomando el papel de "embajadores/as" tanto en el CRPS como en actos externos, buscando la oportunidad de crecer en cada circunstancia. Los objetivos del programa van más allá del mantenimiento o la prevención de recaídas, facilitando los objetivos de las personas que desean incluirse en sociedad y desempeñar su rol de ciudadanos de pleno derecho.

Además, si busca los indicadores de recuperación (Liberman, 2004):

El manejo del malestar. Espacio para la diversión y el bienestar, "olvidándose de la enfermedad"; Conocer las dificultades y superarlas o aprender a convivir con ellas.

El rol significativo. Realizar una actividad significativa que permita la recuperación de roles constructivos y socialmente valiosos. Validando su participación en el programa: opiniones, decisiones. Son los/as protagonistas y el motor del programa.

La promoción de la autonomía. Se favorece la toma de decisiones, sin censurar ideas. Ellos/as preparan las secciones, también fuera de los horarios de radio. Ellos/as asumen el grueso de las relaciones externas: correos, teléfono, congresos.

Las relaciones sociales. Se potencia el trabajo en equipo y la relación personal y apoyo mutuo entre participantes. Es primordial el contacto con otras personas a través de entrevistas y colaboraciones. La actividad promueve la constitución de redes de apoyo y promueve la inclusión social y comunitaria. El programa se realiza en una radio ajena a cualquier organismo o institución.

\section{Vivencias en la radio}

La parte más importante del artículo que nos ocupa será una transcripción (lo más literal posible dentro de las normas gramaticales y semánticas) de un debate generado ad hoc por los/as locutores/as. El tema que quisieron compartir con los/ as posibles lectores/as fue el de su "experiencia en la radio". De esta manera, serán su voz, sus opiniones y sus experiencias personales las que realmente den valor a este escrito.

Los/as locutores/as han permitido que demos sus nombres reales para la redacción de este artículo. Para presentarlos podemos decir que Manuel es el más veterano y Toñi acaba de empezar su andadura con nosotros/as. Fran siempre pone el punto ecológico, mientras que Martín y Javi son los comentaristas deportivos más entregados.

Manuel: Hola, muy buenos días. Vamos a hacer un pequeño coloquio contando nuestra experiencia a lo largo de estos años en las radios que nos acogen desde hace más de 6 años. Haremos 
a hacer una ronda para que cada uno explique más o menos cómo es y ha sido, y cómo le está ayudando este programa que a todos nos une y con el que aprendemos cosas. ¿Qué nos puedes contar, Fran?

Fran: Yo escribí un folio más o menos y os lo voy a leer. Y entonces ya debatimos. Mi experiencia en la radio empezó hace dos años aproximadamente. La primera vez que fui a la emisora no hablé nada al micrófono; estaba interesado, pero a la vez cohibido. Me sorprendió la facilidad con la que mis compañeros decían las cosas y lo veía muy profesional. Sentí que aprendería y que quería probar lo de la radio. Una vez, al principio de estar en la radio, me asignaron moderar el debate y me pareció que mis compañeros dieron mucho esfuerzo y sinceridad y me gustó mucho. Fuera de la emisora, cuando me preguntaban por el grupo de radio, estaba ilusionado y la gente se interesaba por lo que hacía. Me escribieron un e-mail agradeciendo el cuento que leí en la emisora, y lo que me gustó mucho fue el aplauso de mis compañeros. Las visitas que hemos tenido en la radio me han aportado mucho: con unas me lo he pasado bien, me he desahogado, y con otras, me han superado [emocionalmente], pero todas las valoro. A mitad de mi estancia, tuvimos que dar más la cara porque se interesaron por nosotros varios medios de comunicación, como radio, prensa y televisión. Fue emocionante; sin saberlo, hemos aspirado y conseguido mucho. A mi parecer no podemos subir más el nivel, porque a pesar de que los profesionales nos exigen, tampoco es que seamos periodistas. Una anécdota que me gustó mucho fue la de una chica, Lucia, que llamó a la emisora y eso hizo que nos gustara sentirnos escuchados. (Aplausos y Risas).

Manuel: ¿El siguiente? ¡Ataca, Martín!

Martín: Mi experiencia en la radio comenzó hace seis años en Desencadena Usera [primera emisora en la que estuvimos], una radio del barrio. Y me acuerdo que era un pequeño local, sólo había uno o dos micrófonos. De ahí nos fuimos a Onda Merlín Comunitaria, una radio de Villaverde [barrio de Madrid] donde el espacio es grande y hay más micrófonos. En los mandos estaba mi amigo Agustín, al que echo de menos, ya que no sigue con nosotros. Bueno, mis compañeros actuales son Toñi, la única mujer aparte de Itzíar, que está a los mandos; Francisco Javier, Jesús, Manuel, Fran y Óscar, que también está en los mandos. Mi experiencia ha sido positiva, hemos tenido bastantes entrevistas, como a psiquiatras, psicólogas o a un cantante llamado David Celorrio. También a personas sin hogar de Fuenlabrada [ciudad dormitorio de Madrid]. Fuimos invitados por la COPE, que es una radio española. También nos visitó “La 2", de Televisión Española y "Locos por Madrid” [un programa de otra cadena de televisión, con temática variada]. En fin, me gusta lo que hago y espero conocer más gente y que esta nos conozca. Somos personas con enfermedad mental y no queremos que nos excluyan de la sociedad.

Manuel: ¡Bien, otro aplauso! (Aplausos)

Manuel: La señorita de enfrente, que no ha sido la única señorita que ha pasado por aquí. Señorita Toñi...

Toñi: Hola, bueno, yo soy un poco novata en esto, porque estoy grabando desde octubre del año 2010. Mi experiencia en la radio, por lo poco que llevo, es que aprendo cosas, me divierte. También mis compañeros me tratan muy bien y, no sé qué decirte, mi familia está muy contenta con que hable en la radio, me escuchan normalmente.

Fran: Toñi, perdona, ¿qué es lo que más te gusta de la radio?

Toñi: Que me divierto mucho y, aparte, que no tengo queja de mis compañeros, porque yo creo que como soy la única mujer [locutora] me tratan mejor por eso. Creo que aprendes y, bueno, no sólo aprendes, también te lo pasas muy bien. Muchos besos.

Manuel: Aquí llega Javi...

Javier: Bueno, mi trayectoria dentro del grupo de radio ha resultado positiva en progresión, entretenida, divertida y constructiva. Me acuerdo que todo empezó cuando grababa en un ordenador en Los Cármenes [CRPS vinculado al programa de radio] unos apuntes sobre cine y luego se colgaban en un programa resumido de la emisora Desencadena Usera. El programa entonces se emitía en diferido y duraba una hora. La emisora estaba en un centro cultural, con una especie de jardín en el que había un avestruz... Yo participaba poco en la emisión, tenía un miedo espantoso a hablar. Posteriormente nos cambiamos a la emisora actual: ahora el programa de una hora es en directo y también vía Internet. Todos los compañeros que formamos el equipo de radio nos compenetramos y nos ayudamos unos a otros. Con el paso de los años he ido perdiendo poco a poco el miedo a expresarme y he cogido experiencia en todos los sentidos. A lo largo de todos estos años hemos sido reclamados por varios medios de comunicación: prensa, radio, televisión... En los cuales hemos sido capaces de reivindicar nuestra lucha contra el estigma y, al mismo tiempo, expresar en qué consiste nuestro programa, para qué nos sirve, en qué nos ayuda, por qué estamos en este grupo de radio, los objetivos que nos marcamos, etc. También, aprovechando nuestra dinámica de programa, hemos recibido innumerables visitas a la emisora y a nuestro lugar de preparación del programa, en Los Cármenes. Asimismo, hemos entrevistado a todos y todas los que han pasado cerca de nosotros [se refiere a gente que ha pasado como alumnos/as en prácticas de sus distintos estudios]. En definitiva, y en concreto para mí, la radio con el grupo será un motivo más de satisfacción y de mejoría inevitable, espero que por mucho tiempo.

Manuel: Y aquí llega el último y el primero en aterrizar en el programa. Tengo que agradecer al CRPS de Los Cármenes y a sus profesionales, que han hecho mucho y que tarde o temprano van a ir dejando responsabilidades y eso es muy beneficioso para todos los que estamos aquí en la radio. No sólo por el hecho de venir aquí y hablar; en algún momento alguno se pondrá en los mandos, aspecto en el que ahora nos están ayudando, y mucho. Bueno, mi experiencia viene en realidad de mucho tiempo atrás. 
A mí siempre me gustó la radio y cuando yo llegué había veces que me enganchaba tanto que llegaba a tirar la grabadora al suelo. Ahora ya tengo más confianza: lo que ha pasado es ahora un añadido más, porque poco a poco vas haciendo cosas que creías que no podías. Aunque nos cuesta más cocinar [ellos/as elaboran previamente las recetas que van a contar en la sección de cocina], algunas veces nos lanzamos en casa y lo hacemos. También te interesas más por otro tipo de deportes y no sólo por el fútbol; o te interesas por el deporte que se hace en el CRPS. Pasa lo mismo con la cultura, que a veces la dejamos un poco de lado y es muy enriquecedora; te ayuda incluso a retomar la lectura. Además, nosotros mismos damos un toque especial a lo que leemos; damos nuestra opinión: aquí no tenemos cortapisas. Lo soltamos y nos quedamos tan a gusto. Eso sí, procuramos no herir la sensibilidad de nadie. La sección más importante del programa es sin duda el debate o la entrevista. Ha venido gente de Fuenlabrada [ciudad de la comunidad de Madrid]... De muchos sitios. Por todo esto estamos muy agradecidos, porque han visto en nosotros un bastón. Sería muy bonito terminar haciendo una red de radios y que pudiéramos acabar con el estigma hacia las personas con enfermedad mental. Tenemos la facilidad de que desde aquí nos pueden escuchar en todo el mundo; a través de nuestra página Web, del teléfono, etc. En cuanto a mi experiencia personal, puedo contar que hay días en los que no me apetece venir. Pero me motivo pensando que a mí me gusta la radio y que el tiempo que me estoy expresando, luego hace que salga con el pecho hinchado de satisfacción. Eso sí, me gustaría estar en una emisora más grande y que nos pudiera escuchar más gente directamente a través del dial. A ver compañeros. ¿Vosotros qué pensáis a la hora de tener que venir a hacer el programa?

Toñi: Pues mira Manuel, yo suelo hacer mis secciones en casa y traigo mi guión. Además, insisto en que yo me lo pasó muy bien en la radio y pasó un rato muy divertido.

Manuel: Hay que señalar el apoyo que tenemos de todos. Si a alguien no se le ocurre algo, otra persona pide paso y eso hace que todo fluya y salga bien. Valoro mucho a los compañeros cuando les cuestan más algunas secciones y se superan. Yo sé que hay gente que no le gusta el deporte y en cambio hay otros los que les gusta mucho por ejemplo el fútbol. Por ejemplo, Martín puede hablarnos de su experiencia con el equipo de fútbol del CRPS.

Martín: A mí el fútbol me gusta en general y más todavía cuando hay campeonatos entre los centros. Me gusta cuando juego.

Javi: Yo quería decir, respecto a la cocina, que aunque parece ser que a nadie nos gusta cocinar, yo creo que te ayuda: de alguna manera, aprendes. Es una necesidad, porque el día en que estés solo en casa y nadie te haga la comida, pues te ayudará a ser más independiente.

Fran: Yo estoy de acuerdo con Manuel en que venimos los lunes con algo de sueño, con la moral baja, y en cambio sales y sientes que has trabajado en grupo, que hay un orden, mucho respeto por los compañeros y por los profesionales del centro. Parece que así te tomas la semana algo más alegre y muy motivado.

Javier: Es que venir aquí un lunes tan pronto y que lo primero que tengas que hacer sea el programa de radio cuesta un poco.

Manuel: Pero, ¿a qué merece la pena el esfuerzo una vez que te vas a tu casa? Es como si hubiéramos trabajado... En realidad, yo me lo tomo como un trabajo. ¿Cuántas emisoras de radio en los que los locutores cobran pueden encadenar lo que nosotros aquí? A ellos se lo darán todo hecho y sólo tienen que decirlo. Nosotros tenemos el doble de trabajo. ¿A vosotros os parece que en algún momento de vuestra vida esto puede ser un trabajo?

Toñi: Yo no estoy capacitada.

Manuel: Pero si tú, Toñi, desde el primer día lo has hecho con mucha naturalidad, y luego te oye tu familia. Por cierto, ¿qué opináis cuando os escucháis en Internet?

Javier: La verdad es que no me gusta mucho oírme, me noto raro.

Manuel: Realmente es tan natural como cuando de pequeños nos grabábamos en una cinta y decíamos lo que se nos ocurría.

Javier: Yo lo que veo es que es una diferencia muy notable de cuando estábamos en la otra emisora, Desencadena Usera, y allí hacíamos un programa en diferido. Hacerlo ahora en directo requiere mucha atención, mucha más concentración.

Martín: Esto me hace recordar a los compañeros [que ya no están en el programa], por ejemplo Dani. También recuerdo a Diego, de Desencadena Usera.

Javier: Echamos de menos a nuestro compañero Agustín, que se encargaba de los mandos. Lo hacía muy bien, lo controlaba todo, lo hacía de maravilla.

Manuel: También nos acordamos de Rosa y a Julia. Para retomar el coloquio os lanzo una pregunta: ¿Qué os gustaría mejorar?

Javier: Creo que ahora mismo, no es que hayamos llegado al tope, sino que estamos en un momento en que se pueden mejorar muchas cosas, pero pequeños detalles. Lo difícil es mantenerse en el tiempo, como nos hemos mantenido nosotros durante tantos años, porque por este grupo de radio han pasado muchísimas personas. Unas han estado más tiempo y otras menos, y yo creo que mantenernos aquí en el tiempo durante tantos años los compañeros y compañeras que estamos ahora, eso es un motivo de satisfacción para mí.

Manuel: ¿Qué crees que podrías aportar si siguieras durante los próximos años? Aparte de tu inconmensurable esfuerzo.

Javier: Pues, toda la experiencia que he adquirido en este tiempo en la radio. Ahora lo hago un poco mejor, aunque haya 
días en que cometo errores y parece que voy para atrás, pero yo creo que voy en progresión.

Manuel: Toñi, sabemos que aún te cuesta, pero ¿qué crees que te podría ayudar a mejorar de lo que has visto aquí en la radio?

Toñi: Quizá, por el poco tiempo que llevo en la radio, yo no me veo como una gran periodista. Pero sí que me enriquece, aprendo cosas de mis compañeros, sobre todo, aunque yo no hago radio para ser una gran profesional: hago radio para relacionarme y pasármelo bien.

Manuel: Y si supieras que vas a estar aquí al menos otros 40 años, ¿en qué te gustaría evolucionar?

Toñi: Pues por ejemplo, en no tener tanta ayuda de mi familia para preparar alguna sección; poder hacerlo todo por mi cuenta, más fluido y lo mejor posible, pero ya te digo que como una gran periodista no me veo.

Manuel: ¿Y tú, Fran?

Fran: Bueno, es una pregunta difícil para mí, porque a lo mejor pongo ese punto negativo, aunque solo sea un punto. Decir que a lo mejor en mi caso he llegado a estar muy alegre y satisfecho con los resultados que he tenido en estos dos o tres años y parece ser que ahora, pues lo que comentaba Javier, mantenernos en la radio lo veo difícil porque, como dice Toñi, no somos periodistas. Para mí, siempre lo he dicho, esto es una excusa para estar activo. A lo mejor con otra actividad conseguiría los mismos resultados, hay más actividades en el CRPS que no son la radio; pero esta implica para mí más esfuerzo y la considero un buen estímulo para mi rehabilitación. Entonces, para mejorarlo, prefiero seguir con lo que estamos realizando actualmente: compartir experiencias con nuestros compañeros de Portugal [un programa de radio de naturaleza similar al nuestro]; intercambiar archivos con nuestras inquietudes; crear una red, como dejaba caer Manuel al principio, que podría ser una red social y de radio.

Manuel: Martín, cuéntanos tus ideas para mejorar tú o el programa de radio.

Martín: Me gustaría aprender de gente que viene de fuera, que nos cuenta sus experiencias, sus vivencias. Como el Centro de Día de Fuenlabrada [centro para personas sin hogar]. Me impactaron los testimonios de que estaban en la calle, lo duro que era... Para mí la radio es algo que me estimula y que me gusta. Así que pienso seguir.

Manuel: Hay veces que desconocemos los problemas que tiene la gente. A mí me gustaría mejorar la forma en que podemos dirigirnos a las personas que vienen a vernos. Trabajar un poco más ese tema. La verdad es que intentamos hablar con ellos primero de las preguntas que vamos a hacer. También me gustaría mejorar mi ímpetu.
Toñi: Para mí, mi compañero Manuel es muy inteligente, pero a veces muy aturullado.

Martín: Manuel, es que te metes mucho en el asunto. ¡Lo vives!

Manuel: ¡Yo es que la radio la vivo!

Fran: Quería comentaros una cosa. Estuve escuchando ayer un programa en la radio pública y comentaban la intimidad que se tiene y las bases de datos de las empresas; sobre todo la intimidad y el peligro en las redes sociales. Yo llegué a pensar que hemos llegado muy arriba, más de lo que pensaba aquí en Ábrete Camino y, bueno, estamos en Internet. Con esto quiero decir que pueden saber de ti, saber tu opiniones personales y quizá esto puede echarte un poco para atrás. De todas formas, implica un poco "salir del cascarón": por un lado te da un poco de temor pero, por otro, te satisface poder hacer un trabajo y estar en la vida.

Manuel: ¡En definitiva, nos encanta venir aquí!

\section{Conclusiones}

Como se puede apreciar en el diálogo procedente de un debate radiofónico, los/as participantes valoran como positiva la presencia de la radio en sus vidas, influyendo en aspectos tales como las relaciones sociales, el entretenimiento, la adquisición de rutinas, la lucha contra el estigma o la mejora en habilidades sociales (timidez, empatía, asertividad...). Así pues, lo que empezó como un simple grupo más dentro del CRPS Los Cármenes, ha demostrado ser una actividad significativa en la vida de los/as participantes y una iniciativa de probado impacto social, que sólo sería posible con el esfuerzo y entrega de dichos/ as participantes. Además, Ábrete Camino se constituye en una actividad promotora de la integración comunitaria y la promueve, de manera natural, la existencia de redes de apoyo social. No queremos concluir sin recordar que Ábrete Camino es un programa de radio hecho íntegramente por usuarios/as del CRPS de Los Cármenes en Carabanchel y que puede ser escuchado en directo todos los lunes de 10:30 a 11:30 (GMT+1) en Onda Merlín Comunitaria, en el 107.3 FM (si estás en Villaverde), o bien en www.omcradio.org desde cualquier lugar. Se puede participar en directo en el programa llamando al 349150535 29, escribirnos cualquier comentario a abretecamino@yahoo. es, o escuchar resúmenes del programa en www.abretecamino. blogspot.com

\section{Referencias}

Farkas, M. (2007). The vision of recovery today: what it is and what it means for services. World Psychiatry, 6, 4-10.

Liberman, R. P., \& Kopelwicz, A. (2004). Un enfoque empírico de la recuperación definir la recuperación e identificar los factores que pueden facilitarla. Rehabilitación Psicosocial, 1(1), 12-29. 
384 I. M. González, O. P. Pérez, F. J. B. Roca, M. J. Martín, A. R. del Campo, G. S. Rodrígues \& J. F. S. Encabo

Nota:

1. Red Pública de Atención Social a personas con enfermedad mental. Comunidad de Madrid, consejería de Familia y Asuntos sociales. www.madrid.org

Itziar Martínez González é psicóloga e terapeuta ocupacional no Centro de Rehabilitación Psicosocial (CRPS) de Los Cármenes, Madrid, España. Endereço para correspondência: Calle María Martínez 2 (local), 28025, Madrid, España. Teléfone: (91)4621063. E-mail: crpslcarmenes@intress.org. E-mail: trastomg@yahoo.es Óscar Pérez López é Educador Social no Centro de Rehabilitación Psicosocial (CRPS) de Los Cármenes, Madrid, Espanã. E-mail: crpslcarmenes@intress.org. E-mail: educadorcarmenes@gmail.com

Francisco Javier Bueno Roca é usuário do Centro de Rehabilitación Psicosocial Los Cármenes (CRPS), Madrid, España. E-mail: crpslcarmenes@intress.org

Manuel Jiménez Martín é usuario do Centro de Rehabilitación Psicosocial Los Cármenes (CRPS), Madrid, España. E-mail: crpslcarmenes@intress.org

Antonia Ruiz del Campo é usuária do Centro de Rehabilitación Psicosocial Los Cármenes (CRPS), Madrid, España. E-mail: crpslcarmenes@intress.org

Guillermo Segura Rodríguez é usuário do Centro de Rehabilitación Psicosocial Los Cármenes (CRPS), Madrid, España. E-mail: crpslcarmenes@intress.org

Juan Francisco Sigüenza Encabo é usuário Centro de Rehabilitación Psicosocial Los Cármenes (CRPS), Madrid, España. E-mail: crpslcarmenes@intress.org 